\title{
Devastating Sinus Headache Treated with Surgical Drainage in an Adolescent Male
}

\author{
Seul Ah Jeong, MD', Jae Young Cho, MD', Jung Sook Yeom, MD ${ }^{1,2}$ \\ 'Department of Pediatrics, Gyeongsang National University Hospital, Jinju, Korea \\ ${ }^{2}$ Gyeongsang Institute of Health Science, Gyeongsang National University College of Medicine, Jinju, Korea
}

Received: March 7, 2020

Revised: May 2, 2020

Accepted: May 2, 2020

Corresponding author:

Jung Sook Yeom, MD

Department of Pediatrics, Gyeongsang National University

Hospital, Gyeongsang National

University College of Medicine, 79

Gangnam-ro, Jinju 52727, Korea

Tel: $+82-55-750-8338$

Fax: +82-55-752-9339

E-mail: jsyeom@gnu.ac.kr
The sinus headache is a widely accepted diagnosis among patients and primary-care physicians. Contrary to general belief, the cases that fulfilled the International Headaches Society (IHS) criteria for "headache attributed to acute rhinosinusitis" is uncommon [1]. Sinus involvement has not been proven in up to $90 \%$ of self- or healthcare provider-diagnosed sinus headaches, which often satisfy the IHS criteria for migraine [1]. Furthermore, sinus headaches are generally self-limiting [2]. Based on these findings, the relevance of sinus headache has declined steadily in the medical community [2]. However, headache caused by sphenoid sinusitis is a different matter. Sphenoid sinusitis may be a potentially serious disorder, which typically affects preadolescent and adolescent patients and may cause loss of vision, permanent multiple craniopathies, and intracranial spread [3]. Most patients with sphenoid sinusitis present with headache; however, the symptoms are variable [3]. Physical examination, such as palpation of the face, is generally not useful for the detection of sphenoid sinus pathology due to its anatomical location [3]. Therefore, unless pediatric neurologists have a high suspicion of sphenoid sinusitis as the cause of a headache, treatment can be delayed with potentially fatal consequences. Here, we report the case of an adolescent male with severe headache and intractable facial pain that was resistant to medical treatment who recovered immediately after surgical management of sphenoid sinusitis.

A 12-year-old male visited the emergency room with a 7-day history of progressive headache and fever that developed an hour before arrival. On initial assessment, his body temperature was $38.4^{\circ} \mathrm{C}$, the white blood cell count was $11,210 / \mathrm{mm}^{3}$, and his C-reactive protein (CRP) level was $2.8 \mathrm{mg} / \mathrm{L}$ (normal limit, 0 to 5 ). His neurological examination was unremarkable, including no signs of meningeal irritation and normal cerebrospinal fluid examination. He complained severe throbbing headache in the right temporal and occipital regions, sensitivity to light, and nausea. He had no history of the previous headache. Facial tenderness or pain, and post-nasal drip were not observed. The headache was aggravated by not only physical activity but also lying down. Magnetic resonance imaging (MRI) revealed opacity of the right ethmoid sinus, left maxillary sinus, and bilateral sphenoid sinus, but was otherwise normal (Fig. 1). The patient was treated with ampicillin/sulbactam and ketorolac based on the possibility of status migrainosus with acute bacterial infection of the paranasal sinuses. However, after admission, his clinical course progressed rapidly: fever persisted up to $40^{\circ} \mathrm{C}$, his CRP levels increased to $50 \mathrm{mg}$ /

Copyright (C) 2020 Korean Child Neurology Society

This is an Open Access article distributed under the terms of the Creative Commons Attribution Non-Commercial License (http://creativecommons.org/licenses/by-nc/4.0/) which permits unrestricted non-commercial use, distribution, and reproduction in any medium, provided the original work is properly cited. 


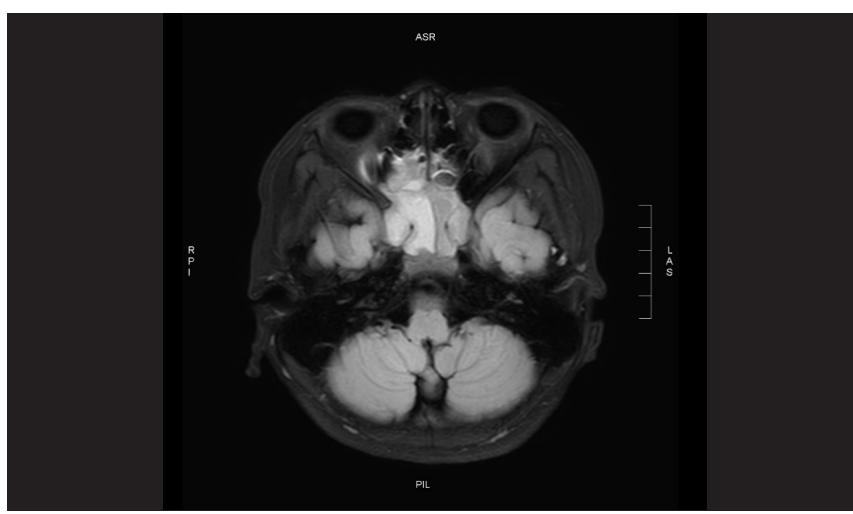

Fig. 1. T2-weighted flair magnetic resonance imaging revealed opacity in the ethmoid and sphenoid sinuses, with more severe involvement of the right side.

$\mathrm{dL}$, and the severity of the headache intensified in the right temporal region of his head. On day 3 of admission, the patient appeared septic and complained of pain in the right retro-orbital region and face. Because the aching facial pain was consistent with the distribution of the trigeminal nerve, we strongly suspected sphenoid sinusitis as the cause of the headache and facial pain. The patient underwent emergency endoscopic surgery and was treated with vancomycin $(40 \mathrm{mg} / \mathrm{kg} /$ day $)$ and metronidazole $(30 \mathrm{mg} / \mathrm{kg} /$ day). A large amount of pus was drained from the right ethmoid and sphenoid sinuses; however, no bacteria was cultured from his specimen. After surgical intervention, all of the symptoms, including the fever, improved rapidly. He was discharged on the 9th day of admission without complications. Written informed consent by the patients was waived due to a retrospective nature of our study.

Our case met the IHS criteria for "headache attributed to acute rhinosinusitis" in terms of the ipsilateral location of the headache (right temporal and retro-orbital regions), purulent material in the right ethmoid and sphenoid sinuses, and the symptoms improved immediately after surgical drainage of the sinuses. Other several symptoms and signs also support sinusitis as the cause of his headache: no previous headache history, progressive headache with fever, worsening pain in lying down position, and the MRI findings of paranasal sinus pathology. Nevertheless, sinus headache was not an initial diagnostic impression of this patient.

Sinusitis rarely causes headache when the sinus in question cannot drain [2]. Purulent nasal discharge is the exception rather than the rule in sphenoid sinusitis [3] The typical location of headache associated with sphenoid sinusitis is the vertex, and the pain may be hemicranial [3]. However, it should be noted that the presence of nasal discharge and headache location in relation to specific sinus structures as signs of sinus headache were omitted from the revised IHS classification system [4]. Conversely, the presence of su- perficial aching hemifacial pain, consistent with the distribution of the trigeminal nerve and retro-orbital pain, are definitive signs of a headache attributable to acute rhinosinusitis, particularly with sphenoid involvement. The sensory innervation of the sphenoid sinus is derived from the ophthalmic and maxillary divisions of the trigeminal nerve. Trigeminal nerve involvement thought to be linked with a headache caused by sinusitis as well as migraine, maybe one reason why the two are easily confused [5]. It is well known that migraines are frequently mistaken for sinus headaches [1]. However, there seems to be a lack of awareness that sphenoid sinusitis is like masquerading as a migraine [5]. We initially suspected migraine, rather than sinus headache, in our patient due to the unilateral headache and photophobia. Unlike migraine, delayed treatment for acute sphenoid sinusitis can cause serious morbidity. The sphenoid sinus is adjacent to several crucial structures that may be affected by sphenoid sinusitis, including cranial nerves II, III, IV, V, and VI; the cavernous sinus; optic canals; dura mater; and the internal carotid artery [3]. The spread of infection to these structures can lead to significant complications [3]. Surgical intervention should be performed immediately in patients; if during the antibiotic therapy, the symptoms get worse or continue for 24 to 48 hours or if there are signs of complications [3]. Our patient's symptoms were intractable to medical treatment but improved immediately after surgical drainage.

Neurologists are generally skeptical of sinus headaches, which may result in their underdiagnosis in clinical practice. However, sphenoid sinusitis may be a potentially devastating disease, and headache is the most common presenting symptom. Our case is a reminder that pediatric neurologists need to have a high index of suspicion when patients with headache took unusual clinical courses of sphenoid sinus involvement.

\section{Conflicts of interest}

No potential conflict of interest relevant to this article was reported.

\section{ORCID}

Seul Ah Jeong, https: / / orcid.org/0000-0002-9597-366X

Jung Sook Yeom, https://orcid.org/0000-0003-0688-7493

\section{Author contribution}

Data curation: SAJ. Formal analysis: SAJ. Methodology: JSY. Visualization: JSY. Writing-original draft: SAJ. Writing-review \& editing: JYC and JSY. 


\section{References}

1. Schreiber CP, Hutchinson S, Webster CJ, Ames M, Richardson MS, Powers C. Prevalence of migraine in patients with a history of self-reported or physician-diagnosed "sinus" headache. Arch Intern Med 2004;164:1769-72.

2. Jones NS. Sinus headaches: avoiding over- and mis-diagnosis. Expert Rev Neurother 2009;9:439-44.
3. Caimmi D, Caimmi S, Labo E, Marseglia A, Pagella F, Castellazzi AM, et al. Acute isolated sphenoid sinusitis in children. Am J Rhinol Allergy 2011;25:e200-2.

4. Headache Classification Committee of the International Headache Society (IHS). The International Classification of Headache Disorders, 3rd edition. Cephalalgia 2018;38:1-211.

5. Ng YT, Butler IJ. Sphenoid sinusitis masquerading as migraine headaches in children. J Child Neurol 2001;16:882-4. 